\title{
Comparison of Maxillary and Mandibular Incisor Inclination in Class II Division 1 Malocclusion among Chinese Children
}

\author{
Dr. Manju Bajracharya \\ Lecturer, Dept of Orthodontics \\ People's Dental College, Kathmandu, Nepal \\ Correspondence: manjub7g@hotmail.com
}

\section{ABSTRACT}

Objective: To determine maxillary and mandibular incisor inclination in Class II Division 1 malocclusion among different vertical skeletal type Chinese children.

Materials \& Method: Lateral cephalograms of Class II Division I malocclusion cases of 120 Chinese children (male-55, female-65) of the age range 12-14 years were divided into three vertical skeletal types (low angle, average angle, high angle) based on Sella-Nasion to Mandibular plane angle. To determine incisor inclination, lateral cephalometric radiographs were measured and analyzed using factorial analysis. All angular parameters were processed using ANOVA and t-test. Maxillary and mandibular incisor inclinations were discussed in detail among three vertical facial skeletal types.

Result: The maxillary and mandibular incisors of low angle individuals were found to be proclined more than those with high and average angle individuals.

Conclusion: Different vertical facial types of Class II Division I malocclusion in early permanent dentition accord to establish discriminate values to set up the cephalometric standards for diagnosis and treatment planning for the clinicians.

Key words: cephalometric radiographs, Class II Division 1 malocclusion, incisor inclination

\section{INTRODUCTION}

Class II Division 1 malocclusion represents the most common skeletal discrepancy seen in orthodontic practice. Understanding the morphology is the key element in treatment planning of this type of malocclusion.' Thus, proper diagnosis of Class II Division 1 malocclusion is essential. Orthodontists have been interested in the multitude of differences in diagnosis and treatment response of various vertical skeletal patterns namely high angle (hyperdivergent), average angle (normal) and low angle (hypodivergent).

The cortical plates of incisal alveolar bone is considered as the anatomic limitations for tooth movement. ${ }^{2-4}$ in non-growing individuals, these anatomic limitations are considered as important factor in assessing the therapeutic effect. The hypothesis of 1:1 cortical bone remodeling verses tooth movement for the retraction of maxillary incisors was tested by Alexander et al in 1998; ${ }^{5}$ as a result they suggested the ratio of 1:2 to assess the prognosis of antero-posterior or postero-anterior movement of maxillary incisors. The attempt of extensive palatal movement of maxillary incisors were done by several investigators. ${ }^{2,3,4,5}$ When roots of these teeth were brought into contact with palatal cortex, it bent and remodeled to some extent but further movement led to cortical plate penetration with subsequent bone loss and root resorption..$^{6-11}$

The objective of this study was to determine maxillary bone inclination in the incisor region of Class II Division 1 malocclusion having different vertical skeletal facial types of Chinese subjects of early permanent dentition, and to investigate the possible differences between Chinese male and female subjects. The study would help in clinical investigation of how much retraction of maxillary incisors is possible in premolar extraction cases of fixed orthodontic treatment procedure.

\section{MATERIALS AND METHOD}

A retrospective study was done with 120 pretreatment lateral cephalogram records of Class II Division 1 malocclusion (55 males and 65 females) aged between 12-14 years of early permanent dentition obtained from Department of Orthodontics, The Affiliated Hospital of Stomatology, 


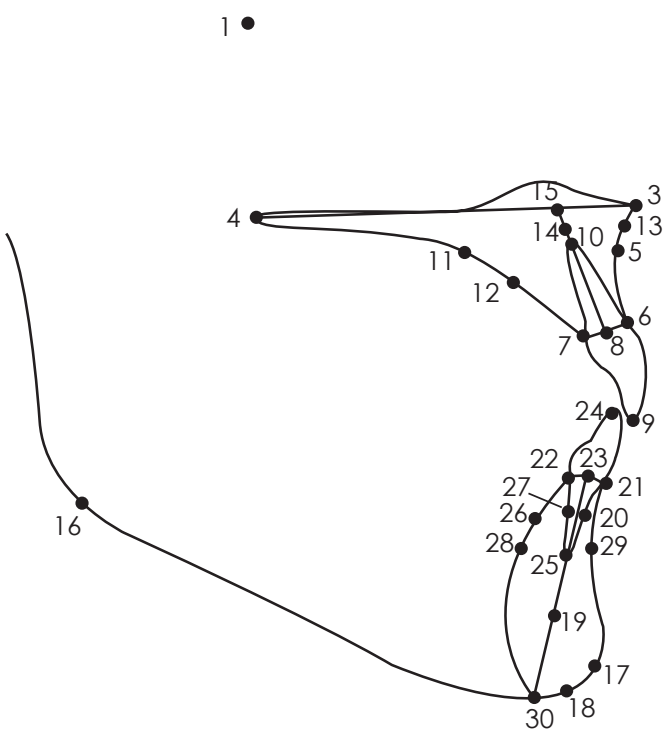

Figure 1: Reference points

Chongqing Medical University, Chongaing, China. The selection was based on Sella-Nasion to Mandibular plane angle (SN-MP angle) that divided the subjects into three vertical skeletal facial types: high angle group (male-12, female 18), average angle group (male-27, female-31) and low angle group (male-16, female-16). The SN-MP angle for high angle group was $>40$ degrees, average angle group was between 29-40 degrees, and low angle group was < 29 degree for Chinese subjects. Inclusion criteria were good quality cephalometric radiographs, presence of all permanent teeth including second molars, Class II molar and canine relationships with Angle's Class II Division 1 malocclusion, overjet $\geq 4 \mathrm{~mm}$ with or without open bite (for high angle subjects). Exclusion criteria were subjects with previous history of orthodontic treatment, any restoration involved in incisal edge of upper and lower central incisors, ectopic eruption of the maxillary and mandibular incisors.

To analyze cephalometric landmarks, lateral cephalomeric radiographs were saved in JPEG format. Anatomical landmarks of 30 points and 14 reference planes were defined according the landmarks described by Akira et $a l^{12}$ Bechmann et $a l,^{13-15}$ Handelmann et $a^{16}$ (Figure 1, 2).

Anatomical points were: 1.A, 2.N, 3.ANS, 4.PNS, 5.Point A, 6.ULP (upper labial alveolar bone margin), 7.UPP (upper palatal alveolar bone margin), 8.UM (maxillary alveolar meatus mid point), 9.UI (upper incisor tip edge), 10.UIR (upper incisor root apex), 11.PA (palatal counterpart of point A), 12.PC (intersection point between a perpendicular to maxillary alveolar axis through point through point A and palatal border of the maxillary frontal midsagittal bone), 13.UCL (upper labial cortical bone of maxilla), 14.UBaM (a center of the rectangle limited by the line A-PA and palatal plane represents the mid sagittal section of basal bone of maxilla), 15.P (intersection between bony hard palate and maxillary alveolar axis, the maxillary alveolar axis runs from the mid point of the alveolar

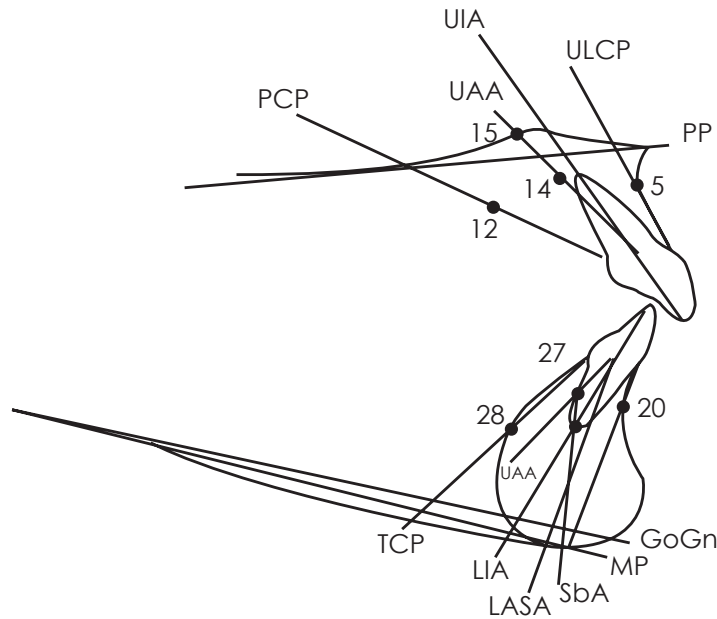

Figure 2: Reference planes

meatus of maxillary central incisors through center point of alveolar meatus), 16.Go, 17.Gn, 18.Me, 19.Point D (the centre point of basal mid saggital bone according to Steiner), 20.Point B, 21.LLP (lower labial alveolar bone margin of central incisors), 22.LTP (lower lingual alveolar bone margin of central incisors), 23.LM (mid point of alveolar meatus of lower central incisors), 24.LI (lower incisor tip edge), 25.L1R (lower central incisors root apex), 26.TB (intersection between a perpendicular to the mandibular axis through point $B$ and lower lingual border of symphysis), 27.LBaM (mid point between B and TB), 28.TC (point on the posterior symphysial border, most distant from the mandibular alveolar axis), 29.LLC (lower labial cortical bone of mandible at symphysial area), 30.Sb (intersection between the lower border of the symphysis and mandibular alveolar axis) (Figure 1).

Anatomical reference planes were: 1.SN plane, 2.PP plane, 3.Mp plane (Down's), 4.GoGn plane (Steiner), 5.UIA plane (upper central incisor long axis by UI, UIR point), 6.UAA (plane connecting points UM-P), 7.ULCP plane (plane connecting points A-ULP), 8.PCP plane (plane connecting points PC-UPP), 9.LIA plane (plane from long axis of lower incisor to points LILIR), 10.LASA plane (plane form Sb and LM), 11.LAA plane (plane from long axis of lower alveolar bone through line connecting points LM to LBaM), 12.SbA plane (plane from point LBaM, D), 13.LLCP plane (plane from point B, LLP), 14.TCP plane (plane axis from point TC to LTP) (Figure 2).

To determine incisor inclination, lateral cephalometric radiographs were measured and analyzed using factorial analysis. Statistical analyses were done using SPSS 10.0 software. Analysis of variance (ANOVA) was performed to access difference among three groups of facial skeletal types. Independent t-test was done to find the difference between the incisor bone inclination within the groups and difference between male and female groups. For all statistical analyses, $p<0.05$ was considered significant. 


\section{RESULT}

Among various vertical skeletal types relating to upper central incisor to cranial base, angle ULCP-UIA in male individuals showed statistical significant difference. High angle individuals showed more negative inclination than low and average angle individuals (Table 1). Also, female of high angle individuals showed more negative inclination than low and average angle individuals (Table 2).

Table 1: t-Test of significance of ULCP-UIA angle in each vertical skeletal types of male subjects

\begin{tabular}{|c|c|c|c|c|c|c|c|c|c|}
\hline \multirow{2}{*}{$\begin{array}{l}\text { Angular measurements } \\
\text { (in degree) }\end{array}$} & \multicolumn{3}{|c|}{$\begin{array}{l}\text { Average angle male } \\
\qquad \mathrm{N}=27\end{array}$} & \multicolumn{3}{|c|}{$\begin{array}{l}\text { High angle male } \\
\qquad \mathrm{N}=12\end{array}$} & \multicolumn{3}{|c|}{$\begin{array}{l}\text { Low angle male } \\
\qquad=16\end{array}$} \\
\hline & Mean & SD & $p$-value & Mean & SD & $p$-value & Mean & SD & $p$-value \\
\hline ULCP-UIA & -6.18 & 4.07 & 0.8 & -9.87 & 6.77 & $0.03^{*}$ & -7.58 & 9.18 & 0.10 \\
\hline
\end{tabular}

* Statistically significance at $p<0.05$

Table 2. t-Test of significance of ULCP-UIA angle in each vertical skeletal types of female subjects

\begin{tabular}{|c|c|c|c|c|c|c|c|c|c|}
\hline \multirow{2}{*}{$\begin{array}{l}\text { Angular measurements } \\
\text { (in degree) }\end{array}$} & \multicolumn{3}{|c|}{$\begin{array}{l}\text { Average angle male } \\
\qquad N=31\end{array}$} & \multicolumn{3}{|c|}{$\begin{array}{l}\text { High angle male } \\
\mathrm{N}=18\end{array}$} & \multicolumn{3}{|c|}{$\begin{array}{l}\text { Low angle male } \\
\qquad \mathrm{N}=16\end{array}$} \\
\hline & Mean & SD & $p$-value & Mean & SD & $p$-value & Mean & SD & $p$-value \\
\hline ULCP-UIA & -5.98 & 6.22 & 0.89 & -6.28 & 5.62 & $0.03^{*}$ & -3.47 & 3.95 & 0.109 \\
\hline
\end{tabular}

* Statistically significance at $p<0.05$

According to the present study, angles PCP-UIA, UAA-UIA, SN-PCP, SN-UIA, SN-ULCP, SN-UAA were more in low angle individuals compared to average and high angle individuals. There was no statistical difference in male and female subjects combined together whereas individually male and female subject showed statistical significant difference. Angles LLCP-LIA, GoGn-TCP showed more in low angle male and female as compared to average and high angle male and female subjects; while angles GoGn- LIA, GoGn-LLCP, GoGn-LAA were more in average angle male and female subjects compared to low and high angle male and female. Similarly, angle GoGn-SbA showed more in high angle as compared to average and low angle male and female subjects. Also, angle TCP-LIA showed more negative inclination in low angle compared to high and average angle male and female samples and angle LASA-LIA showed more negative in average angle compared to low and high angle male and female individuals (Table 3, 4).

Table 3: t-Test of significance in maxillary incisor jaw bone measurements of combined subjects in each vertical skeletal types

\begin{tabular}{|c|c|c|c|c|c|c|c|c|c|}
\hline \multirow{2}{*}{$\begin{array}{l}\text { Angular measurements } \\
\text { (in degree) }\end{array}$} & \multicolumn{3}{|c|}{$\begin{array}{l}\text { Average angle male } \\
\qquad N=59\end{array}$} & \multicolumn{3}{|c|}{$\begin{array}{l}\text { High angle male } \\
\qquad \mathrm{N}=29\end{array}$} & \multicolumn{3}{|c|}{$\begin{array}{l}\text { Low angle male } \\
\qquad \mathrm{N}=32\end{array}$} \\
\hline & Mean & SD & $p$-value & Mean & SD & $p$-value & Mean & SD & $p$-value \\
\hline PCP- UIA & 18.83 & 7.29 & 0.13 & 15.41\# & 7.3 & 0.18 & 20.89\# & 9.76 & 0.53 \\
\hline UAA-UIA & 2.64 & 5.81 & 0.4 & 2.7 & 6.73 & 0.34 & 4.67 & 7.11 & 0.38 \\
\hline SN-PCP & $124.99 *$ & 6.81 & 0.52 & $117.01^{*}$ & 13.59 & 0.37 & $130.99 *$ & 6.9 & 0.8 \\
\hline SN-UIA & 106.44 & 7.07 & 0.36 & 105.06 & 10.58 & 0.5 & 110.46 & 11.47 & 0.71 \\
\hline SN-ULCP & 98 & 6.92 & 0.2 & 95.34\# & 6.04 & 0.32 & 100.32\# & 6.84 & 0.68 \\
\hline SN-UAA & 109.10* & 4.59 & 0.9 & $104.62 *$ & 5.7 & 0.62 & $113.50^{*}$ & 5.72 & 0.78 \\
\hline PCP- UIA & 18.83 & 7.29 & 0.57 & 15.41\# & 7.3 & 0.88 & 20.89\# & 9.76 & 0.8 \\
\hline UAA-UIA & 2.64 & 5.81 & 0.4 & 2.7 & 6.73 & 0.34 & 4.67 & 7.11 & 0.38 \\
\hline
\end{tabular}

Statistically significance at $p<0.05$ 
Table 4: $t$-Test of significance in mandibular incisor jaw bone measurements of combined subjects in each vertical skeletal types

\begin{tabular}{|c|c|c|c|c|c|c|c|c|c|}
\hline \multirow{2}{*}{$\begin{array}{c}\text { Angular measurements } \\
\text { (in degree) }\end{array}$} & \multicolumn{3}{|c|}{$\begin{array}{c}\text { Average angle male } \\
\text { N=59 }\end{array}$} & \multicolumn{3}{|c|}{ High angle male } & \multicolumn{3}{|c|}{$\begin{array}{c}\text { Low angle male } \\
\text { N=32 }\end{array}$} \\
\cline { 2 - 11 } & Mean & SD & p-value & Mean & SD & p-value & Mean & SD & p-value \\
\hline LLCP-LIA & $5.09 \&$ & 3.34 & 0.3 & $5.01 \& \#$ & 4.39 & 0.36 & $5.89 \#$ & 6.2 & 0.15 \\
\hline TCP-LIA & $-3.93 @$ & 5.38 & 0.06 & $-1.99 \#$ & 3.76 & 0.58 & $-7.28 @ \#$ & 4.64 & 0.17 \\
\hline GoGn-TCP & $105.02 \&$ & 8.95 & 0.65 & $98.75 \# \&$ & 8.82 & 0.84 & $108.37 \#$ & 7.58 & 0.13 \\
\hline GoGn-LIA & 100.3 & 6.47 & 0.92 & 95.46 & 6.84 & 0.7 & 93.73 & 24.9 & 0.69 \\
\hline GoGn-LLCP & 94.16 & 6.61 & 0.78 & 93.24 & 6.88 & 0.87 & 93.76 & 7.71 & 0.95 \\
\hline GoGn-LAA & $99.00 \&$ & 6.25 & 0.82 & $91.74 \&$ & 16.76 & 0.89 & 97.18 & 14.98 & 0.9 \\
\hline GoGn-SbA & $89.29 \&$ & 5.86 & 0.86 & $93.07 \# \&$ & 5.98 & 0.65 & $89.76 \#$ & 5.56 & 0.85 \\
\hline D & 9.25 & 3.61 & 0.83 & 13.65 & 2.86 & 0.34 & 10.8 & 4.38 & 0.36 \\
\hline LASA-LIA & -10.36 & 3.84 & 0.88 & -8.67 & 3.63 & 0.67 & -10.24 & 3.67 & 0.56 \\
\hline
\end{tabular}

Statistically significance at $p<0.05$

\#: there is difference between high angle and low angle groups

@: there is difference between average angle and low angle groups

\&: there is difference between high angle and average angle groups

*: there is difference between high angle, low angle and average angle groups

\section{DISCUSSION}

The retrospective study was performed to identify the maxillary and mandibular incisal inclination among different vertical skeletal types. The difference in the incisal jaw inclination of Class II Division 1 malocclusion among three vertical skeletal types between gender groups were discussed in detail. The anatomic limits of labial and lingual cortical plates of incisor apex may be regarded as "orthodontic walls". ${ }^{16}$ In order to compensate sagittal and vertical discrepancies, there must be sufficient of alveolar bone to move the incisors in desired direction, but how much movement of incisors were possible without crossing these anatomic limits were unknown. Since Class II Division 1 malocclusion is phenotype characterized by abnormalities in both dentolaveolar and skeletal discrepancy, only trying to compensate skeletal discrepancy through alveolar remodeling could not succeed in biological ground, ${ }^{17}$ orthognathic surgery may be required for stable occlusion, soft tissue harmony and improvement of skeletal relationship. In this study also, relating to PCP-UIA plane, angle PCP-UIA was more in low angle compared to high angle male and female but in Sun wei's ${ }^{18}$ study this angle showed no statistical significance difference.

Relating to SN plane, angle SN-PCP showed higher in low angle than average and high angle of Class II Division 1 malocclusion which was also agreed to Sun Wei et al ${ }^{18,19}$ result, the anterior cranial bone relation to upper incisor plane axis showed more in low angle than high angle male and female. The bone inclination to upper labial cortical plane axis (ULCP) of low angle individual (100.32 \pm 6.84$)$ inclined more lingually than high angle male and female subjects (95.34 \pm 6 .04). Angle SN-UAA angle was also high in low angle individual than average and high angle subjects.

Palatal bone inclination from ANS-PNS to PCP and UAA plane axis were also more in low angle than high and average angle male and female, which agreed with Sun Wei et al ${ }^{18}$ result. The inclination between lower labial cortical plate axis to lower incisor axis (LLCP-LIA) mean value showed less in high angle as compared to average and low angle male and female subjects. It demonstrated that high angle individuals exhibit less compensation inclination than average and low angle individuals. Also, angle TCP-LIA was in less negative inclination in high angle compared to average and low angle. Angle GoGn-TCP was higher in low angle than average and high angle male and female suggesting the compensation inclination in low angle male and female subjects were more than high and average angle male and female. However angle GoGn-LAA exhibited more in average angle than high angle cases while angle GoGn-SbA was more in high angle compared to low and average angle cases in this study result. Angle LASA-LIA showed positive inclination which was also more in low angle than high angle male and female subjects.

\section{CONCLUSION}

As per the inclination relating to SN plane and GoGn plane; the incisors and alveolar bone of maxillary and mandibular incisor jaws inclined more labially in low angle than high angle 
individuals of Class II Division 1 malocclusion. So, Clinicians can move the incisors more labially in low angle individuals than high angle individuals.

Among different vertical facial types of Class II Division 1 malocclusion in early permanent dentition, the ability of compensation may be stronger in maxillary than mandibular incisors area. The clinicians should pay more attention when moving the incisors root apex of maxilla and mandible towards the palatal and lingual side as the region between point PA, PC on the maxillary cortical plate, TB, TC on the mandibular cortical plates may be considered as borderline for palatal and lingual movement of incisors for prevention of cortical plate perforation.

\section{OJN}

\section{REFERENCES}

1. Nanda R. Biomechanics and esthetic strategies in clinical orthodontics. Elsvier. 2005.

2. Edwards JG. A study of the anterior portion of the palate as it relates to orthodontic therapy. Am J Orthod Dentofac Orthop. 1976; 69:249-173.

3. Ten Hoeve A, Mulie RM. The effect of the anterioposterior incisor repositioning on the palatal cortex as studied with laminography. J Clin Orthod. 1976; 10:804-22.

4. Mulie RM, Ten Hoeve. The limitations of tooth movement within the symphysis studied with laminography and standardized occlusal films. J Clin Orthod. 1976; 12: 882-899.

5. Alexander D. Vardimon, Eyal Oren, Yocheved Ben-Bassat. Cortical bone remodeling/tooth movement ratio during maxillary incisor retraction with tip versus torque movements. Am J Orthod Dentofac Orthop. 1998; 14:520-9.

6. Duterloo HS: The impact of orthodontic treatment procedures on the remodeling of the alveolar bone. J Orthod studie Ned Ver Ortho. $1975 ; 66: 5-21$.

7. Wainwright W.M. Faciolingual tooth movement: its influence on the root and cortical plate. Am. J Orthod Dentofac Orthop. 1973; 64:278302.

8. Remmelink HJ, Van der Molen AL. Effects of anterioposterior incisor repositioning on the Root and Cortical plate: A Follow-up study. J Clin Orthod. 1984; 18:42-49

9. Reitan K. Influence of variation in bone type and character on tooth movement on different alveolar bone types. Angle orthod. 1964 3:244-55.

10. Kurol J, Thilander B. Infraocclusion of primary molars and the effect on occlusal development, a longitudinal study. Eur J. Orthod. 1984; 6:277-93.

11. Murakami T, Yokota S, Takahama Y. Periodontal changes after experimentally induced intrusion of the upper incisors in Macaca fuscata monkeys. Am J Orthod Dentofac Orthop. 1989; 95:115-26.

12. Akira H, Hitoshi H, Kazuhide K. Correlation between cortical plate proximity and apical root resorption. Am J Orthod Dentofac Orthop. 1998; 114:311-8.

13. Bechmann SH, Segner D. Changes in the alveolar morphology during open bite treatment and presiction of treatment results. Eur J Orthod 2002; 24:391-406.

14. Bechmann SH, Kuitert R, B, Prahl- Andersen B, Segner D. The RPS and tainzing DB, Alveolar and skeletal dimensions associated with lower face height. Am J Orthod Dentofacial Othop. 1998; 113:498-506.

15. Bechmann SH, Kuitert RB, Prahl- Andersen B, Segner D. The RPS and Tainzing SB. Alveolar and skeletal dimensions associated with overbite. Am J Orthod Dentofacial Othop. 1998; 113:443-52.

16. Handelman CS, The anterior alveolus: Its importance in limiting orthodontic treatment and its influences on the occurrence of iatrogenic sequele. Angle Orthod. 1996; 66:95-110.

17. Murray C, Meikle MC. The dentomaxillary complex and overjet correction in class II division 1 malocclusion. Am J Orthod Dentofac Orthop. 1980; 2:184-197.

18. Sun Wei, Zhou Li.A cephalometric study of incisal jaw morphology of normal occlusion subjects (unpublished Dissertation). Hua xi kou qiang yi xue Za Zhi. (in Chinese) 2005.

19. Sun Wei I Zhou Li, Bai D, Zhao MY. K-cluster analysis of incisal jaw morphology of normal occlusion subjects among different vertical skeletal types. Hua xi kou qiang yi xue Za Zhi. 2005 Aug; 23(4):299-302,309. 